\title{
Optimization of Critical Data Synchronization via Link Overlay RNG in Mobile Ad Hoc Networks
}

\author{
Emmanuel Baccelli, Juan Antonio Cordero and Philippe Jacquet \\ E-mails: Emmanuel.Baccelli@inria.fr, cordero@lix.polytechnique.fr, Philippe.Jacquet@inria.fr \\ INRIA Saclay - Île-de-France \\ Parc Orsay Université, 4 rue J. Monod, 91893 Orsay Cedex, France
}

\begin{abstract}
In practice, ad hoc networks are still too unreliable for standard mobile and vehicular communications. It is thus important to complement current protocols in this context, with schemes guaranteeing the exchange of critical data when needed. A promising approach in this realm is to use an overlay subgraph, over which critical messages are exchanged and acknowledged in a peer to peer fashion. Overlay nodes' local databases remain thus synchronized over time, at least concerning critical data. This paper elaborates on the problem of performance, related to the discovery and maintenance of such overlay networks in a mobile ad hoc context. We analyze SLOT, an overlay selected based on a Relative Neighbour Graph (RNG) scheme. We then apply SLOT to a standard IP protocol: OSPF, a popular routing protocol which has recently been extended, with RFC 5449 and RFC 5614, to work also on mobile ad hoc networks, and which makes use of a similar overlay synchronization subgraph. This paper compares the performance of these existing OSPF mechanisms with that of SLOT-OSPF, a novel OSPF extension for mobile ad hoc networks using SLOT. Simulations show that SLOT-OSPF produces drastically less control traffic than RFC 5449 or RFC 5614, allowing SLOT-OSPF to function correctly while the other existing approaches stall, when the number of routers in the domain is large.

Index Terms - ad hoc; routing; OSPF; MANET; IP; IETF; MPR; mobile; evaluation;
\end{abstract}

\section{INTRODUCTION}

Mobile ad hoc networks are currently not practical for standard mobile and vehicular communications. One of the main reasons for this is the substantial lack of reliability that still plague ad hoc communications to date. In particular, guaranteed dissemination of critical data, whether it is control data or application data, is a problem that is ignored by most existing ad hoc networking solutions. It is thus important to complement existing ad hoc broadcast mechanisms with a parallel scheme which guarantees the dissemination of critical data when necessary. The most promising schemes in that regard are those involving an overlay network, i.e. a mobile ad hoc network subgraph, over which critical messages are acknowledged hop-by-hop, in a peer to peer fashion, in order to verify the actual dissemination, or, absent expected acknowledgement, retransmit the corresponding critical message. Such an approach ensures that overlay nodes' local databases remain synchronized over time, at least concerning critical data.
This paper elaborates on the problem of performance, related to the discovery and maintenance of such overlay network in a mobile ad hoc context. Related work in this domain mainly include MPR schemes [4] and connected dominating set (CDS) schemes such as [26] and [32]. This paper analyzes the theoretical performance of SLOT (Synchronized Link Overlay - Triangular), an overlay selected based on a Relative Neighbor Graph (RNG) scheme. SLOT operation is defined in two modes: cost-based and ID-based. These two versions of the SLOT scheme are analyzed and compared in terms of link set size and stability.

The paper then applies this overlay technique (in IDbased mode) to a standard IP protocol: Open Shortest Path First (OSPF [1][2]), a popular routing protocol which incorporates such a local database synchronization scheme for critical control data. OSPF has recently been extended by the IETF with RFC 5449 ([10], based on MPR) and RFC 5614 ([27], based on CDS) to operate on ad hoc networks of moderately mobile nodes without stringent memory or power constraints. Operating OSPF on ad hoc networks is a seducing idea for at least two reasons. On one hand because of legacy: OSPF is an extremely well deployed, known, and renowned, protocol from the IP suite. On the other hand, because of unification: operating OSPF on ad hoc networks gathers wired and wireless IP networking under a single routing solution - an interesting perspective industry-wise, in terms of maintenance and costs.

This paper confirms the results produced by the theoretical analysis with simulations of the MANET extension of OSPF, comparing the performance of RFC 5449 and RFC 5614 with that of SLOT-OSPF, an alternative OSPF extension for operation on MANETs using a SLOT overlay instead of the MPR overlay used by RFC 5449 or the CDS employed by RFC 5614. The paper concludes that SLOT-OSPF produces drastically less control traffic than RFC 5449 or RFC 5614, which allows SLOT-OSPF to function correctly while RFC5449 and RFC 5614 stall, when the number of routers (and thus the router density) in the domain grows larger. 


\section{A. Related Work}

Initial work on OSPF extension for wireless ad hoc networks by [12], [13] paved the way for early protocol design such as [20] and [31] for OSPFv2. Building on this ground work [7] compared the performance of two approaches for OSPFv3 operation on MANETs: Overlapping Relays with Smart Peering (OR/SP) and MANET Designated Routers (MDR). Later, [9] and [21] studied and compared the performance of another approach for OSPFv3, MPR-OSPF (based on Multi-Point Relays, i.e. MPR) with that of OR/SP. Work in this domain has lead to IETF standardization, for instance RFC 5449 and RFC 5820 ([10] [11], both based on MPR schemes), and RFC 5614 ([27], based on an MDR scheme). This paper aims at improving over this state of the art, by analyzing the performance of a novel approach, SLOT-OSPF, and by comparing it with the existing approaches. SLOT-OSPF uses overlay techniques inspired from [24]. Extensive prior work exists in the domain of overlays for ad hoc networks, including for instance [32] and [26] on connected dominating sets, [28] on weakly connected dominating sets, or [30] on overlays based on multi-point relaying.

\section{The SLOT AlgORITHM}

The goal of this section is to analyze the performance of a scheme selecting an overlay network in a mobile ad hoc context. This overlay network is to be used to synchronize critical data among the different nodes in the ad hoc network.

\section{A. Requirements}

Since the mobility of these nodes can be substantial and ad hoc communication resources are generally scarce, it is essential to select an overlay network that fits best the following targets:

1) Low overlay link density;

2) Low overlay link change rates.

Low overlay link density is paramount because a high density results in a huge number of acknowledgement and data packets (re)transmissions. For example, let's consider a high density network with $N$ nodes, having each $M$ neighbor, on average. If the overlay network were the full network, then the transmission of a single critical message will generate a quadratic $N M$ number of acknowledgements and packet transmissions. Experiments with standard OSPF show that wifi based networks cannot support more than 20 nodes [20] without collapsing due to packet retransmissions and acknowledgement cascade, if the overlay network is the whole network.

Low overlay link change rate is a critical consequence of node mobility and wireless medium variations. It is however not necessarily a simple function of the overlay link density. As we will see in this paper, it is possible to have an overlay $S_{1}$ with a lower overlay link density than another overlay $S_{2}$, while the latter generates less overlay link changes. Nevertheless, if the overlay network is the whole network and if the average link lifetime is $T$, then the new overlay link creation rate will be $\frac{M N}{T}$, which is the rate of basic peer to peer database synchronization.

In this section we introduce a scheme, SLOT, which identifies and maintains an overlay network, based on Relative Neighbor Graph (RNG [17] [24], itself inspired from the Gabriel graph [16], except RNG does not need positioning devices, only link cost estimates). A subset of the whole set of links network-wide, are selected to be part of the overlay network. Links can be selected according to link quality, in order to reduce the packet loss and the cost of data synchronization. It is indeed anticipated that future radio networks will operate on on-line programmable radio-interface which will naturally increase the throughput when nodes are closer. In this case the cost metric can be a function increasing with the distance.

We will first specify the SLOT scheme, and then evaluate it analytically with a model based on unit disk graph and random walk mobility in two dimensions. We will then generalize these results for other dimensions.

\section{B. Algorithm Details}

Since synchronization happens symmetrically on overlay links, these links must of course be bidirectional. In this context, if $m(A, B)$ is the metric on the symmetric link $(A, B)$, we then have $m(A, B)=m(B, A)$. Let's for instance take $m(A, B)=\min \{\operatorname{Cost}(A, B), \operatorname{Cost}(B, A)$, where $\operatorname{Cost}()$. is a specific metric (such as bit delivery delay, remaining bandwidth, financial cost).

A symmetric link $(A, B)$ belongs to the overlay except if there exists a chain of links $\left(A, C_{1}\right), \ldots,\left(C_{i}, C_{i+1}\right), \ldots$, $\left(C_{k}, B\right)$ such that:

- The $C_{i}$ are all neighbors of both $A$ and $B$. - The cost of the links,
$m\left(A, C_{1}\right), \ldots, m\left(C_{i}, C_{i+1}\right), \ldots, m\left(C_{k}, B\right), \quad$ are all
smaller to $m(A, B)$.

In case of link cost equality, the following tie breaking is used: the link with lower cost is the link with the minimum node ID. In case this rule still does not tie the break (because the two links have their minimum ID node in common), then the considered links are part of the overlay.

SLOT is defined as a simplification of the above, considering only triangular relations: a link $(A, B)$ is not selected as part of the overlay if and only if there exist a common neighbor node $C$ such that $m(A, B) \geq \max \{m(A, C), m(C, B)\}$. This can be used when the metric satisfies the triangular inequality: $m(A, B) \leq m(A, C)+m(C, B)$. 
When the link have all the same (uniform) cost, in this case a link $(A, B)$ is removed from the overlay when:

- there exist a node $C$ neighbor to both $A$ and $B$;

- node $C$ ID is smaller to both node $A$ and node $B$ ID's.

In this case the elimination is equivalent to a triangle elimination: the link holding the two largest node ID is eliminated. Fig. 1 shows two examples of SLOT overlay, one with cost based on distance and the second with uniform cost, compared to the total number of network links, with the unit disk graph model.

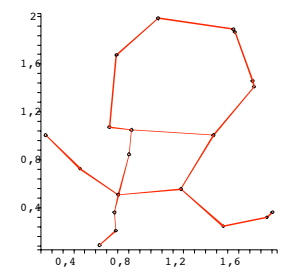

(a) SLOT with link cost (b) SLOT based on IDs based on distance.

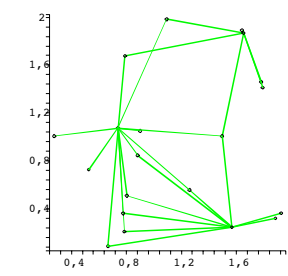
(uniform link cost).

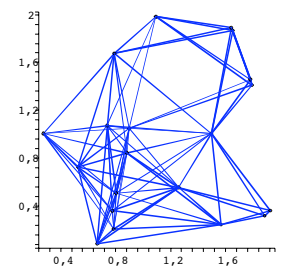

(c) Full network.

Fig. 1. Overlay links, for a network with 20 nodes on a $2 \times 2$ map.

\section{ANALysis of SLOT in Dimension 2}

This section provides a theoretical performance of the SLOT algorithm. In the following we consider the unit-disk graph model of uniform node density $\nu$ and a plan of area $\mathcal{A}$. Therefore the average node neighborhood size is $M_{f}=\pi \nu$. We assume that each node follows an independent random walk equivalent to an average speed of $s$. We also assume that the random walk is isotropic, so that the stationary probability that a node is in a portion of the map of area $\sigma$ and has speed in a cone of aperture $\theta$ is exactly $\frac{\theta \sigma}{2 \pi \mathcal{A}}$. We will consider the asymptotic case where $\nu \rightarrow \infty$ and $\mathcal{A} \rightarrow \infty$. In a first phase we will analyze the performance of SLOT in a two dimension vector map. In a second phase we generalize to other dimensions $D$ : a linear map $(D=1)$ and a cubic map $(D=3)$.

\section{A. General Properties}

Let's observe that the mobility model is sufficiently general. We assume that the nodes move at a constant speed $s$ on a random distance $L$ and then change direction. When $L$ is large, assume $L=\infty$ on an infinite map, this is equivalent to a kind of random waypoint mobility model. When $L \rightarrow 0$ then it is equivalent to a Brownian motion model. However all the independent random walk models have in common the following properties [19].

1) The number of points in a portion of map of size $b$ is a Poisson distribution of density $b \nu$.

2) The rate $f(B)$ at which nodes enter the portion $B$ is proportional to the node density, the length $\ell$ of the border and the average speed of the mobile, that is $f(B)=\frac{\ell s}{\pi} \nu$.

3) If we consider that the portion of the map $B$ also moves like a mobile node, then the rate at which mobile nodes enter the mobile area becomes $\frac{\ell \Delta(s)}{\pi} \nu$ where $\Delta(s)$ is the average relative speed between two random mobile nodes.

Note that if the nodes move at constant speed $s$ (no randomness), then we get $\Delta(s)=\frac{4}{\pi} s$, otherwise the formula is more complicated.

\section{B. Full Network Overlay}

In this section we investigate the specific case where the overlay network is in fact the whole network. The rate at which nodes enter a node neighborhood is $V_{f}=2 \Delta(s) \nu$. We have thus proven the following theorem:

Theorem 1: The average number of links departing from a node is equal to $M_{f}=\pi \nu$ and the average rate of new links per second is equal to $V_{f}=2 \Delta(s) \nu$. If nodes move at the same speed $s$ we have $V_{f}=\frac{8}{\pi} s \nu$.

\section{SLOT Based on Distance}

In this section we investigate the case where the overlay is determined by the SLOT algorithm based on the distance cost metric. Let's begin with a straightforward theorem.

Theorem 2: The graph of the SLOT overlay based on distance is planar.

Proof: This is a well known property of RNG [17], if two links cross over, then it is easy to see that one at least will be eliminated in the SLOT algorithm.

Theorem 3: The average number of overlay links departing from a node in the SLOT algorithm with cost based on distance is equal to [18

$$
M_{d}=\frac{\pi}{2 \frac{\pi}{3}-\frac{\sqrt{3}}{2}}+O\left(\nu e^{-\left(\frac{2 \pi}{3}-\frac{\sqrt{3}}{2}\right) \nu}\right)
$$

and the average rate of new synchronized links from a node is equal to

$$
\begin{aligned}
V_{d}= & \frac{4}{3 \sqrt{\pi}}\left(2 \frac{\pi}{3}-\frac{\sqrt{3}}{2}\right)^{-\frac{3}{2}} \Delta(s) \sqrt{\nu} \\
& +O\left(\Delta(s) \nu^{2} e^{-\left(\frac{2 \pi}{3}-\frac{\sqrt{3}}{2}\right) \nu}\right)
\end{aligned}
$$

Remark: For constant uniform speed $s$ it comes $V_{d}=$ $\sqrt{\pi} \frac{8}{3}\left(2 \frac{\pi}{3}-\frac{\sqrt{3}}{2}\right)^{-\frac{3}{2}} s \sqrt{\nu}$ 
Proof: Let's consider a link between two nodes $A$ and $B$ at distance $r$ of each other. Let $B(r)$ be the intersection of the disks of radius $r$ respectively centered on $A$ and $B$. The condition at which the link belongs to the overlay is that $B(r)$ contains no other nodes than $A$ and $B$. The area of this intersection is $|B(r)|=r^{2} A\left(\frac{\pi}{3}\right)$ with $A(\theta)=2 \theta-\sin (2 \theta)$. Therefore the probability that link $(A, B)$ is included in the overlay is $\exp \left(-\nu r^{2} A\left(\frac{\pi}{3}\right)\right)$.

The average number of links from a random node $A, M_{d}$, that belong to the overlay will be

$$
\begin{aligned}
M_{d}= & \int_{0}^{1} 2 \pi \nu r d r e^{-r^{2} A\left(\frac{\pi}{3}\right) \nu} \\
= & \int_{0}^{\infty} 2 \pi \nu r d r e^{-r^{2} A\left(\frac{\pi}{3}\right) \nu} \\
& +O\left(\nu e^{-\nu|B(1)|}\right) \\
= & \frac{\pi}{2 \frac{\pi}{3}-\frac{\sqrt{3}}{2}}+O\left(\nu e^{-\nu|B(1)|}\right) \\
\approx & 2.557530242+O\left(\nu e^{-\nu|B(1)|}\right)
\end{aligned}
$$

The constant $M_{d}$, Devroye's constant, is known from [18].

For the rate change, the computation is also relatively straightforward. Let's consider a link $(A, B)$ which belongs to the overlay. $B(r)$, the intersection of the corresponding disks of radius $r$, is empty. Therefore the rate at which the link will disappear from the overlay is equal to the rate at which the mobile nodes will enter $B(r)$. Let's call $\partial B(r)$ the border of $B(r)$ and its length $|\partial B(r)|=\frac{4}{3} \pi r$. The entering rate is therefore $|\partial B(r)| \frac{\Delta(s)}{\pi} \nu$. Therefore the rate $V_{d}$ at which overlay links vanish, from a random node $A$, is

$$
\begin{aligned}
V_{d}= & \frac{4}{3} \Delta(s) \int_{0}^{1} 2 \pi \nu^{2} r^{2} d r e^{-r^{2} A\left(\frac{\pi}{3}\right) \nu} \\
= & \frac{4}{3} \Delta(s) \int_{0}^{\infty} 2 \pi \nu^{2} r^{2} d r e^{-r^{2} A\left(\frac{\pi}{3}\right) \nu} \\
& +O\left(\nu^{2} e^{-\nu|B(1)|}\right) \\
= & \frac{4}{3 \sqrt{\pi}}\left(A\left(\frac{\pi}{3}\right)\right)^{-\frac{3}{2}} \Delta(s) \sqrt{\nu}+O\left(\nu^{2} e^{-\nu|B(1)|}\right)
\end{aligned}
$$

Notice that $V_{d} \approx 3.471762654 \times s \sqrt{\nu}$ when the speed is constant. The rate at which links appear is also $V_{d}$.

\section{SLOT with Uniform Link Cost}

In this section we investigate the case where the overlay is determined by the SLOT algorithm based on IDs (uniform link cost).

Theorem 4: The average number of overlay links departing from a node in the SLOT algorithm with uniform link cost, when $\nu \rightarrow \infty$ satisfies

$$
M_{u}=\int_{\frac{\pi}{3}}^{\frac{\pi}{2}} \frac{8 \pi \sin 2 \theta}{2 \theta-\sin 2 \theta} d \theta+O\left(\frac{1}{\nu}\right)
$$

with $A(\theta)=2 \theta-\sin 2 \theta$ and the average rate of new synchronized links from a node tends to

$$
V_{u}=32 \Delta(s) \int_{\frac{\pi}{3}}^{\frac{\pi}{2}} \frac{\theta \sin 2 \theta}{(2 \theta-\sin 2 \theta)^{2}} d \theta+O\left(\frac{1}{\nu}\right)
$$

Remark: When the speed is constant we have $\lim _{\nu \rightarrow \infty} V_{u}=\frac{128 s}{\pi} \int_{\frac{\pi}{3}}^{\frac{\pi}{2}} \frac{\theta \sin 2 \theta}{(2 \theta-\sin 2 \theta)^{2}} d \theta$.
Proof: Let's assume that node $A$ has ID equal to $x$, and $B$ has ID $y$. We consider that the link $(A, B)$ belongs to the overlay and $y>x$ (this will cover half the cases). Assume that the distance between node $A$ and node $B$ is $r$ and let $S(r)$ be the intersection of the disks of radius 1 , respectively centered on $A$ and $B$, i.e. the location of the common neighborhood of $A$ and $B$. Since $(A, B)$ is an overlay link there is no node in $S(r)$ with ID smaller than $\min \{x, y\}=x$.

Since we consider only ID comparison, there is no loss of generality to assume that the IDs of the nodes are scalar numbers uniformly distributed on the unit interval.

The probability that (i) $y>x$ and (ii) the link $(A, B)$ belongs to the overlay is $(1-x) \exp (-\nu x|S(r)|)$. Since $|S(r)|=A(\theta)$ with $r=2 \cos \theta$. Counting also the case where $y<x$ we get

$$
\begin{aligned}
M_{u}(\nu) & =2 \int_{0}^{1} d x \int_{0}^{1} 2 \pi \nu(1-x) r d r e^{-x A(\theta) \nu} \\
& =\int_{0}^{1} \int_{\frac{\pi}{3}}^{\frac{\pi}{2}} 8 \pi \nu(1-x) \sin 2 \theta d \theta e^{-x A(\theta) \nu} \\
& =\int_{\frac{\pi}{3}}^{\frac{\pi}{2}} \frac{8 \pi}{\nu(A(\theta))^{2}} \sin 2 \theta d \theta\left(\nu A(\theta)-1+e^{-\nu A(\theta)}\right)
\end{aligned}
$$

Therefore:

$$
\begin{aligned}
M_{u}(\nu) & =\int_{\frac{\pi}{3}}^{\frac{\pi}{2}} \frac{8 \pi}{A(\theta)} \sin 2 \theta d \theta+O\left(\frac{1}{\nu}\right) \\
& =M_{u}+O\left(\frac{1}{\nu}\right)
\end{aligned}
$$

with

$$
M_{u}=\int_{\frac{\pi}{3}}^{\frac{\pi}{2}} \frac{8 \pi \sin 2 \theta}{2 \theta-\sin 2 \theta} d \theta \approx 3.603973720
$$

To get the rate at which overlay links vanish in the uniform cost algorithm, we just have to consider the same link $(A, B)$ such that nodes are at distance $r$ and their IDs are respectively $x$ and $y$. We assume $x<y$. The area $S(r)$ contains no node with ID smaller than $x$. The rate at which the link $(A, B)$ will disappear as overlay link is equal to the rate at which nodes with ID smaller than $x$ will enter the area $S(r)$. This rate is equal to $|\partial S(r)| \frac{\Delta(s)}{\pi} \nu x$. Since $|\partial S(r)|=4 \theta$ with $r=2 \cos \theta$, the rate at which overlay links disappear (including the case $y<x)$ is:

$$
\begin{aligned}
V_{u}(\nu)= & \Delta(s) \int_{0}^{1}(1-x) x d x \\
& \times \int_{\frac{\pi}{3}}^{\frac{\pi}{2}} 32 \nu^{2} \theta \sin 2 \theta d \theta e^{-\nu A(\theta) x} \\
= & \Delta(s) \int_{\frac{\pi}{3}}^{\frac{\pi}{2}} \frac{32 \theta \sin 2 \theta}{\nu(A(\theta))^{3}} \\
& \times\left(A(\theta) \nu-2+(2+\nu A(\theta)) e^{-\nu A(\theta)}\right) d \theta \\
= & V_{u}+O\left(\frac{\Delta(s)}{\nu}\right)
\end{aligned}
$$

with

$$
V_{u}=32 \Delta(s) \int_{\frac{\pi}{3}}^{\frac{\pi}{2}} \frac{\theta \sin 2 \theta}{(2 \theta-\sin 2 \theta)^{2}} d \theta
$$

When the speed is a constant $s$, we have $V_{u}=\frac{128 s}{\pi} \int_{\frac{\pi}{3}}^{\frac{\pi}{2}} \frac{\theta \sin 2 \theta}{(2 \theta-\sin 2 \theta)^{2}} d \theta \approx 4.146111863 \times$ 


\section{ANALYSis For Other Dimensions}

In this section we extend our previous analysis to the cases where the networks are embedded in a space vector of dimension $D$. So far we have analyzed the case $D=2$. We will analyze the other cases $D=1$ and $D=3$ and give a qualitative generalization for further dimensions.

\section{A. 1-Dimensional Networks}

In this section we investigate the one-dimensional case, which is related to a mobile network on a lane or an infinite straight road. In this case the positions of the nodes are ordered on the real axis as an increasing sequence $\left\{x_{i}\right\}_{i \in Z}$.

Theorem 5: The SLOT graph with distance link cost is made of the links $\left(x_{i}, x_{i+1}\right)$ provided that $x_{i+1}-x_{i}<1$.

Consequently we have the following theorem

Theorem 6: With distance link cost, the average number of SLOT links per node is

$$
M_{d}=2+O\left(e^{-\nu}\right)
$$

and the average overlay link change rate is

$$
V_{d}=2 \Delta(s) \nu+O\left(\nu \Delta(s) e^{-\nu}\right)
$$

Remark: When the speed is constant we have $\delta(s)=s$. since

Proof: These results could come directly from theorem 5

1) The node at position $x_{i}$ is linked to the nodes at position $x_{i-1}$ and $x_{i+1}$, except when those are at distance greater than 1, which happens with probability of order $e^{-\nu}$.

2) A change in those links happens only when the left node (at position $x_{i-1}$ ) or the right node (at position $x_{i+1}$ ) crosses over with another node. A node crosses over another node with rate $\Delta(s) \nu$ and therefore the link rate change of node at position $x_{i}$ is $2 \Delta(s) \nu$.

However, as an exercise, we can derive these results from our previous methodology inspired from the $D=2$ analysis.

The set $B(r)$ is an interval of length $|B(r)|=r$, therefore

$$
\begin{aligned}
M_{d} & =\int_{0}^{1} \exp (-\nu|B(r)|) \nu 2 d r \\
& =2-2 e^{-\nu}
\end{aligned}
$$

In dimension 1, the rate of entrance in a set $B$ is $|\partial B| \frac{s}{2} \nu$ when the flow is isotropic with average motion speed $s$. Indeed $|\partial B(r)|=2$ since $B(r)$ is an interval. Therefore the rate at which overlay links disappear is

$$
\begin{aligned}
V_{d} & =\int_{0}^{1} \int_{0}^{1} 2 \nu d r e^{-\nu|B(r)|}|\partial B(r)| \nu \frac{\Delta(s)}{2} \\
& =2 \Delta(s) \nu-2 \Delta(s) \nu e^{-\nu}
\end{aligned}
$$

Regarding the SLOT algorithm with uniform link cost, we have the following theorem:

Theorem 7: With SLOT uniform, the average number of overlay link per node is

$$
M_{u}=4 \log 2+O\left(\frac{1}{\nu}\right)
$$

The average overlay link change rate is

$$
V_{u}=2 \Delta(s)+O\left(\frac{\Delta(s)}{\nu}\right)
$$

Proof: Here we work with the set $S(r)$ the intersection of the neighborhood of two nodes at distance $r$ away: $|S(r)|=$ $2-r$. In this case we have

$$
\begin{aligned}
M_{u} & =2 \int_{0}^{1}(1-x) d x \int_{0}^{1} e^{-\nu|S(r)| x} 2 \nu d r \\
& =4 \log 2-\frac{2}{\nu}+O\left(e^{-\nu}\right)
\end{aligned}
$$

Regarding overlay link changes we have $|\partial S(r)|=2$ and

$$
\begin{aligned}
V_{u} & =2 \int_{0}^{1}(1-x) x d x \int_{0}^{1} e^{-\nu|S(r)| x} 2 \nu^{2}|\partial S(r)| \frac{\Delta(s)}{2} d r \\
& =2 \Delta(s)-\frac{3 \Delta(s)}{\nu}+O\left(\Delta(s) e^{-\nu}\right)
\end{aligned}
$$

\section{B. 3-Dimensional Networks}

Here we need the following geometric results: $S(r)$ denoting the intersection of unit spheres whose centers are at distance $r$ apart $(r \leq 1)$, we have

$$
\begin{aligned}
|S(r)| & =2 \int_{0}^{1-r / 2} \pi\left(1-\left(\frac{r}{2}+x\right)^{2}\right) d x \\
& =2 \pi\left(\left(1-\frac{r}{2}\right)-\frac{1}{3}\left(1-\frac{r}{2}\right)^{3}\right)
\end{aligned}
$$

and

$$
\begin{aligned}
|\partial S(r)| & =4 \pi \int_{0}^{1-r / 2} d x \\
& =4 \pi\left(1-\frac{r}{2}\right)
\end{aligned}
$$

We also have $|B(r)|=r^{3}|S(1)|$ and $|\partial B(r)|=r^{2}|\partial S(1)|$.

Regarding mobility model the average entrance rate in a volume $B$ is equal to $|\partial B| \frac{s}{4} \nu$ with mobile nodes moving at average isotropic speed $s$.

Furthermore if nodes move at constant isotropic speed $s$, then $\Delta(s)=\frac{4}{3} s$.

Theorem 8: The overlay link per node density for SLOT overlay based on distance link cost in dimension 3 is:

$$
M_{d}=\frac{4 \pi}{3}\left(\frac{12}{11 \pi}\right)^{\frac{1}{3}}+O\left(e^{-11 \pi \nu / 12}\right)
$$

and the per node overlay link change rate

$$
V_{d}=2 \pi^{2}\left(\frac{12}{11 \pi}\right)^{\frac{5}{3}} \frac{1}{3} \Gamma\left(\frac{5}{3}\right) \Delta(s) \nu^{\frac{1}{3}}+O\left(\nu e^{-11 \pi \nu / 12}\right)
$$

Proof: We take the straightforward methodology developed so far and apply it to $D=3$. It comes

$$
\begin{aligned}
M_{d} & =\int_{0}^{1} \exp (-\nu|B(r)|) 4 \pi r^{2} \nu d r \\
& =\int_{0}^{1} \exp \left(-\nu r^{3}|S(1)|\right) 4 \pi r^{2} \nu d r \\
& =\frac{4 \pi}{3|S(1)|^{1 / 3}}\left(1-e^{-\nu|S(1)|}\right)
\end{aligned}
$$

And the overlay link change rate

$$
\begin{aligned}
V_{d}= & \int_{0}^{1} \exp (-\nu|B(r)|) 4 \pi r^{2} \nu d r|\partial B(r)| \frac{\Delta(s)}{4} \nu \\
= & \nu^{\frac{1}{3}} \int_{0}^{\nu^{1 / 3}} e^{-|V(1)| x^{3}} \pi x^{4}|\partial S(1)| \Delta(s) d x \\
= & \nu^{\frac{1}{3}}\left(O\left(e^{-\nu V(1)}\right)\right. \\
& \left.+\int_{0}^{\infty} e^{-|V(1)| x^{3}} \pi x^{4}|\partial S(1)| \Delta(s)\right) d x \\
= & \pi|\partial S(1)||S(1)|^{-\frac{5}{3}} \frac{1}{3} \Gamma\left(\frac{5}{3}\right) \Delta(s) \nu^{\frac{1}{3}} \\
& +O\left(\nu^{\frac{1}{3}} e^{-\nu|S(1)|}\right)
\end{aligned}
$$


For the SLOT overlay based on uniform link cost we have:

Theorem 9: The per node link density with the SLOT algorithm based on uniform link cost is:

$$
\begin{aligned}
M_{u}= & 64 \log (11)-160 \log (2)+ \\
& +64 \sqrt{3}\left(\operatorname{Arctanh}\left(\frac{\sqrt{3}}{6}-\operatorname{Arctanh}\left(\frac{\sqrt{3}}{3}\right)\right)\right. \\
& +O\left(\frac{1}{\nu}\right)
\end{aligned}
$$

and the per node overlay link change:

$$
\begin{aligned}
V_{u}= & \left(-8 \log (2)-\frac{24}{11}+8 \log (11)\right. \\
& +16 \sqrt{3}\left(\operatorname{Arctanh}\left(\frac{\sqrt{3}}{6}-\operatorname{Arctanh}\left(\frac{\sqrt{3}}{3}\right)\right)\right. \\
& \left.+O\left(\frac{1}{\nu}\right)\right) \Delta(s)
\end{aligned}
$$

Proof: We have the new expression

$$
\begin{aligned}
M_{u} & =2 \int_{0}^{1}(1-x) d x \int_{0}^{1} \exp (-\nu|S(r)| x) 4 \pi r^{2} \nu d r \\
& =8 \pi \int_{0}^{1} \frac{\nu|S(r)|-1}{\nu|S(r)|^{2}} r^{2} d r+O\left(e^{-\nu \mid S(0)}\right) \\
& =8 \pi \int_{0}^{1} \frac{1}{|S(r)|} r^{2} d r+O\left(\frac{1}{\nu}\right)
\end{aligned}
$$

and

$$
\begin{aligned}
V_{u}= & \int(1-x) x d x \\
& \times \int_{0}^{1} \exp (-\nu|S(r)| x) 2 \pi r^{2} \nu d r|\partial S(r)| \Delta(s) \nu \\
= & \int_{0}^{1} 2 \pi r^{2}|\partial S(r)| \frac{\nu|S(r)|-2}{\nu|S(r)|^{3}} d r+O\left(\Delta(s) e^{-\nu|S(0)|}\right) \\
= & \int_{0}^{1} 2 \pi|\partial S(r)| r^{2} \frac{1}{|S(r)|^{2}} d r \Delta(s)+O\left(\frac{\Delta(s)}{\nu}\right)
\end{aligned}
$$

\section{AnAlysis Summary}

Table I] summarizes our analysis of SLOT based on distance link cost, and of SLOT based on uniform link cost. For SLOT based on distance link cost, the link density increases with dimension number, but the link change rate decreases. Clearly a generalization to higher dimension would show a link change rate in $O\left(\nu^{\frac{1}{D}}\right)$. With SLOT based on uniform link cost on the other hand, the density and change rates attain their maximum value for $D=2$ and then decrease. The qualitative explanation of this phenomenon is still an open problem.

\begin{tabular}{|r|ll|}
\hline & SLOT Distance \\
\hline$D$ & $M_{d}$ & $V_{d}$ \\
\hline 1 & 2 & $\nu \Delta(s) \times 2$ \\
\hline 2 & 2.557530242 & $\sqrt{\nu} \Delta(s) \times 2.726716013$ \\
\hline 3 & 2.944205854 & $\nu^{\frac{1}{3}} \Delta(s) \times 1.018993779$ \\
\hline
\end{tabular}

\begin{tabular}{|r|ll|}
\hline & SLOT Uniform & \\
\hline$D$ & $M_{u}$ & $V_{u}$ \\
\hline 1 & 2.772588722 & $\Delta(s) \times 2$ \\
\hline 2 & 3.603973720 & $\Delta(s) \times 3.603973720$ \\
\hline 3 & 2.5046958 & $\Delta(s) \times 1.441903356$ \\
\hline
\end{tabular}

TABLE I

RECAPITULATION OF THE ANALYSIS OF SLOT BASED ON DISTANCE LINK COST (TOP TABLE) AND UNIFORM LINK COST (BOTTOM TABLE), FOR DIMENSIONS 1, 2 AND 3.

\section{Vi. Maple Simulations}

Before the SLOT-OSPF simulations presented in the next section, we present some preliminary Maple simulations of the SLOT overlay algorithm on its own, with the unit disk graph model. We measured link density and link creation rate, in a $6 \times 6$ map, with the number of nodes in the network varying from a few to several hundreds. Note that by link density we mean the average number of link departing from a random node.

Figure 2 shows the performance of the two SLOT strategies, namely with distance cost and uniform cost, compared to the full network link density. Some discrepancies between these simulations and theory can be noticed, mainly due to the fact that the simulations run on a finite size map, while the theoretical results hold on infinite map. Indeed, even in a rather "big" $6 \times 6$ map, more than $55 \%$ of the nodes are neighbors of the border, which impacts neighborhood size as well as the probability to have triangular adjacencies. The theoretical results can thus be seen as a theoretical upper bound for the finite size networks that were simulated. There are less border effects with SLOT distance than with SLOT uniform, because SLOT distance is more concerned about nodes close-by. Regarding the latter, the preliminary simulations show results well below the theoretical performance. We also simulated maps of $2 \times 2$ and $4 \times 4$, and as the simulated map grows bigger, the results seem to converge nevertheless towards the theoretical upper bound. Note that the border effects can also be observed on the density of links in full network, where a gap is visible between theory and the simulations.

The mobility model used in these preliminary Maple simulations is the following: nodes move independently according to a random walk with a constant speed of one unit per second. The nodes change direction every 0.01 second. When a mobile node encounter the border it bounces as in a billiard, the outcoming speed vector being the mirror image of the incoming speed vector. We measured the new overlay link creation rate in this context - which is, of course, equal to the average overlay link failure rate in order to have a constant average density.

Figure 3(b) shows the performance of the two SLOT strategies, compared to the full network new link formation rate. We no longer experience a significant gap between theory on infinite map and simulation on finite maps. Notice that the distance cost SLOT is outperformed by uniform cost SLOT, since the latter has a link change rate independent of density while the former has a link change rate depending on the square root of density.

\section{SLOT OVERLAYS APPLIED TO OSPF}

In the following, we will use the SLOT algorithm to provide a synchronization overlay for OSPF on ad hoc networks. We will first give a brief overview of the OSPF protocol, and the 


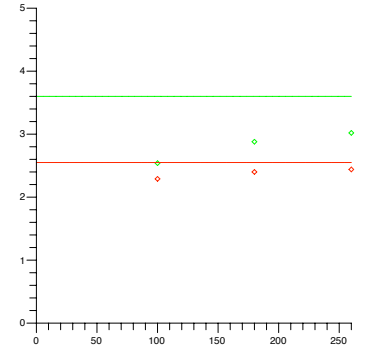

(a) SLOT overlay.

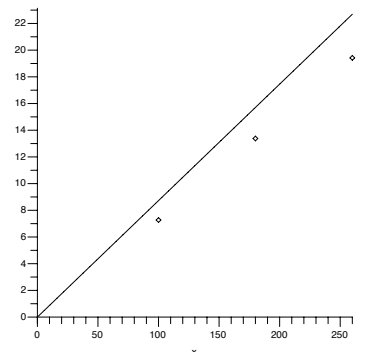

(b) Full network.
Fig. 2. Average link density on a $6 \times 6$ map with Maple simulations (dots), theory (plain), SLOT with distance cost (red), uniform cost (green).

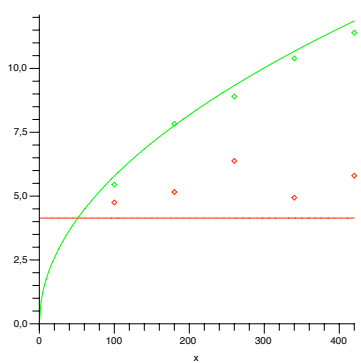

(a) SLOT overlay.

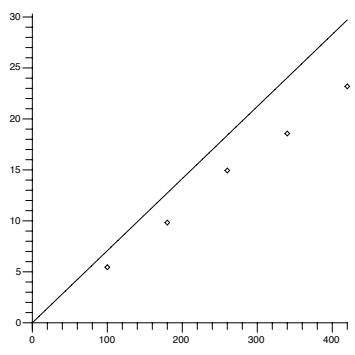

(b) Full network.
Fig. 3. Average per node new link creation rate with speed 1 unit distance per time unit, $6 \times 6$ map. Maple simulations (dots), theory (plain), SLOT with distance cost (green), uniform cost (red).

way SLOT is inserted in this framework - defining SLOTOSPF. We will then present simulation results, that compare the performance of RFC5449 with that of SLOT-OSPF in a mobile ad hoc network context.

\section{A. OSPF on Ad Hoc Networks}

As a proactive link-state routing protocol, OSPF [1] [2] employs periodic exchanges of control messages to accomplish topology discovery and maintenance: Hellos are exchanged locally between neighbors to establish bidirectional links, while LSAs reporting the current state of these links are flooded (i.e. diffused) throughout the entire network. This signaling results in a topology map, the link state database $(L S D B)$, being present in each node in the network, from which a routing table can be constructed. An additional mechanism, particular to OSPF, provides explicit pairwise synchronization of the LSDB between some neighbors, via additional control signaling (database description messages and acknowledgements). Such neighbor pairs are then called adjacent neighbors, while other bidirectional neighbors are called 2-WAY.

Note that the subgraph consisting of the links between adjacent neighbors (i.e. adjacencies) is basically a critical data synchronization overlay network for specific control data (i.e. LSAs). In a wireless ad hoc environment, limited

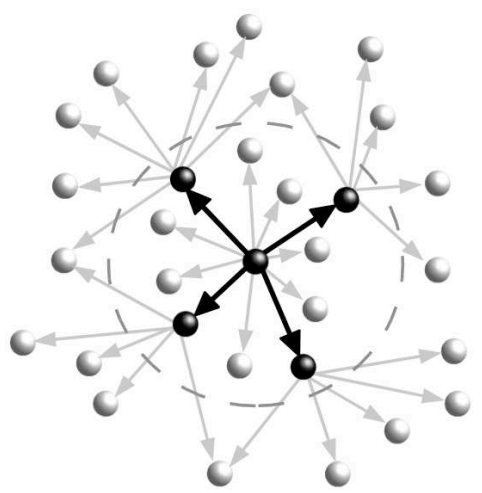

Fig. 4. Multi-Point Relaying (MPR). The center node selects sufficient relays (in black), to cover every node two hops away. Selected relays are then called MPRs. The dashed circle is the radio range of the center node.

bandwidth and interferences between neighbors call for a significant reduction of standard OSPF control traffic [6]. At the same time, router mobility requires Hello and LSA periods to be drastically shortened in order to be able to track topology changes, implying heavier control traffic, without even more efficient control traffic reduction techniques. Moreover, standard OSPF mechanisms such as the Designated Router mechanism are not functional in most ad hoc network scenarii.

RFC 5449 [10] and RFC 5614 [27] thus specify alternative OSPF mechanisms that allow its operation on MANETs. RFC 5449 is based on multi-point relay (MPR) techniques (see Figure VII-A and [4]), while RFC 5614 is based on connected dominating set techniques similar to [26]. The MPR overaly is a special case of connected dominating set overlay, both techniques aiming at reducing the number of forwarders of a given disseminated packet, while still ensuring that this packet is sent to each router in the network. These techniques identify a backbone of relays that is sufficient to cover the whole network. Three main categories of alternative OSPF mechanisms are thus introduced by RFC 5449 and RFC 5614:

- Flooding Optimization and Backup. Instead of the usual, naive flooding scheme, use MPR (RFC 5449) or CDS (RFC 5614) flooding, which reduces redundant retransmissions.

- Adjacency Selection. Instead of attempting to become adjacent (i.e. synchronized) with all neighbors, a router synchronizes only with neighbors in the MPR (RFC 5449) or CDS (RFC 5614) overlays.

- Topology Reduction. In its LSAs, a router reports only links with neighbors part of the MPR (RFC 5449) or CDS (RFC 5614) overlay, instead of all the links.

\section{B. SLOT-OSPF}

SLOT-OSPF is a refinement of RFC 5449, which uses the same framework, and the same mechanisms for flooding 
optimization, backup, and topology reduction (see Section VII-A). However, instead of using the MPR overlay for adjacency selection, SLOT-OSPF uses the overlay produced by the SLOT algorithm described in Section II-B. In other words, adjacencies are formed and maintained only along links that are selected to be part of the SLOT overlay.

\section{OSPF Simulations}

Simulation results shown in the following were obtained based on the Zebra OSPF implementation [14] running on $802.11 \mathrm{~b}$, simulated with the GTNetS [5] simulator. We used the random waypoint model at an average speed of $2,5 \mathrm{~m} / \mathrm{s}$ (in fact $5 \mathrm{~m} / \mathrm{s}$ with pauses of $40 \mathrm{sec}$ ), and we simulated CBR user data UDP flows that amount to a total of $1 \mathrm{Mbps}$, shared among all nodes. Implementation of RFC 5449 follows the IETF specification [10] and the RFC 5614 code is available in [25]. The code for SLOTOSPF and for RFC 5449 is available [15]. See the Appendix for more details on the simulations and simulation parameters.

We observe in Fig. 5 that while the delivery ratio and the end-to-end delay achieved by SLOT-OSPF remains stable when the number of nodes and the node density in the network grow (we simulated up to 100 nodes), RFC 5449, and then RFC 5614 suffer a significant drop in performance. This drop can be explained by the explosion of the amount of control overhead due to RFC 5449 or RFC 5614 operation.

The following focuses on the comparison between SLOTOSPF and RFC 5449. As shown in Fig. 6 both in terms of sheer size and in terms of number of transmitted control packets, RFC 5449 control traffic increases drastically with the size of the network. The control overhead due to SLOT-OSPF operation grows too with the number of nodes in the network, but at a far more reasonable pace. In fact, the amount of overhead due to RFC 5449 or RFC 5614 operation quickly saturates the available bandwidth and when the density increases as the number of node grows over 75 , this saturation makes it more difficult to deliver packets and thus impossible to establish the necessary adjacencies, as shown by the drop in Fig. 7 This makes sense at first sight, as the amount of overhead produced by RFC 5449, for a network with 75 nodes, makes a random node have to bear roughly a local traffic $t_{\text {local }}=\frac{\pi r^{2}}{l^{2}}\left(t_{\text {control }}+t_{\text {data }}\right)$, where $r$ is the radio range $(150 \mathrm{~m}), l$ is the length of the simulated field $(400 \mathrm{~m})$, while $t_{\text {control }}$ is the total control traffic and $t_{\text {data }}$ the total traffic due to user data. In Fig. 6, we measured $t_{\text {control }}$ to be 2,96 Mbps, for 75 nodes. In the same context, we measured the average path length to be 1,7 hops. Since the injected data traffic is $1 \mathrm{Mbps}$, this corresponds to $t_{\text {data }}$ being 1,7 Mbps. We therefore get a $t_{\text {local }}$ of approximately 2 Mbps, reaching standard $802.11 \mathrm{~b}$ saturation throughput [22] [23].
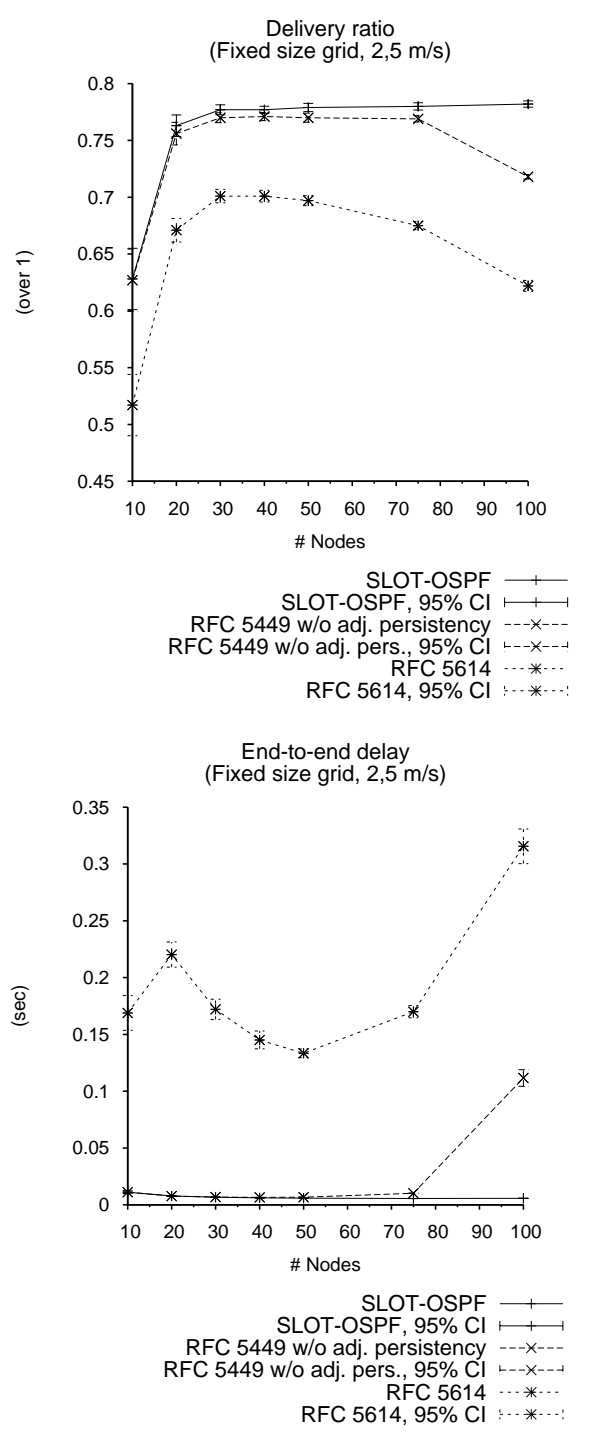

Fig. 5. Delivery ratio and delay in seconds. CBR user data flows, $1 \mathrm{Mbps}$. Fixed size grid, $2,5 \mathrm{~m} / \mathrm{s}$ velocity.

\section{Perspectives ANd Conclusion}

In this paper, we presented and analyzed the performance of SLOT, a scheme based on Relative Neighbour Graphs, which provides a backbone overlay over which critical data can be synchronized between neighbors, in a mobile ad hoc context. We have shown that SLOT produces an overlay made of a number of links per node that is independent of the network density and of the topology (with the unit disk graph model), while the total number of non-overlay link is proportional to the node density, i.e. $O(\nu)$. Typically, an additional synchronization process takes place every time a new link appears in the SLOT overlay. We have shown that with uniform link cost, SLOT provides a per node synchronization rate that is independent of the node density, and proportional to the average node speed $s$. When the cost is on the other hand based on distance, the synchronization rate increases to be $O(s \sqrt{\nu})$, which nevertheless remains 


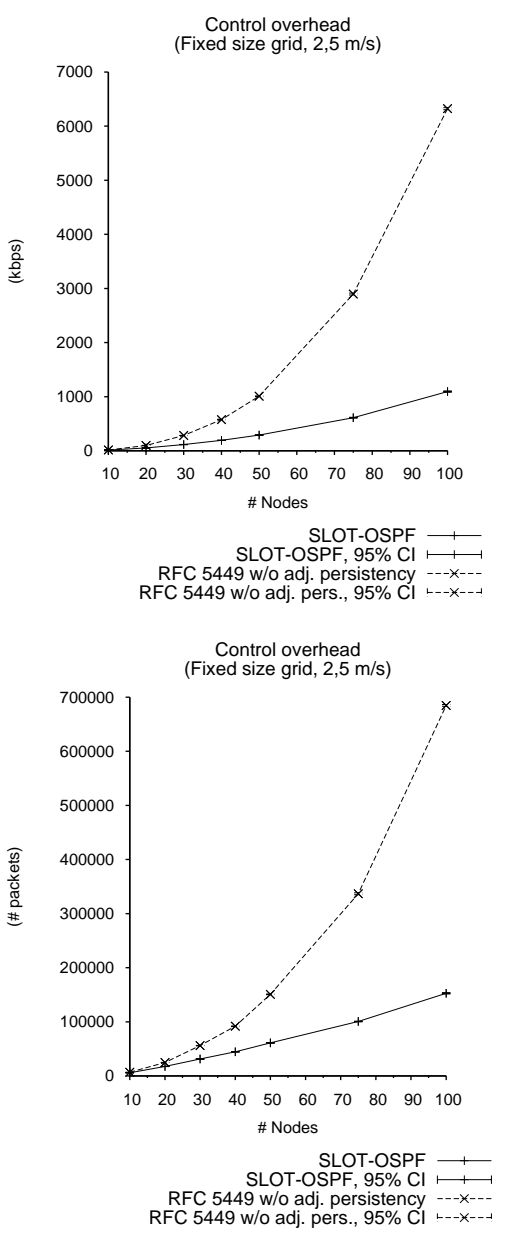

Fig. 6. Control overhead in kbps and in number of packets.

drastically lower than the total link change rate that is $O(\nu s)$. These properties are interesting, as it is highly desirable to have both a small number of links in the synchronization overlay, and a low overlay link change rate.

We have then applied SLOT to a typical IP protocol which uses such critical data synchronization overlay: the routing protocol OSPF, a traditional routing protocol which has recently been extended to work also on mobile ad hoc networks. We have compared, via simulations with $802.11 \mathrm{~b}$, the performance of RFC 5449 and RFC 5614 (OSPF's extensions for ad hoc networks) with that of SLOT-OSPF, the OSPF extension we proposed in this paper, using SLOT with uniform link cost. The simulations show that SLOT-OSPF produces drastically less control traffic than RFC 5449 or RFC 5614 which are based on alternative synchronization overlays (respectively MPR and CDS). This allows SLOT-OSPF to function correctly as RFC5449 and RFC 5614 stall, when the density and/or number of routers in the domain is large.

We expect however that for real deployments, SLOT with cost based on link quality will perform better for synchronization processes than SLOT with uniform cost.

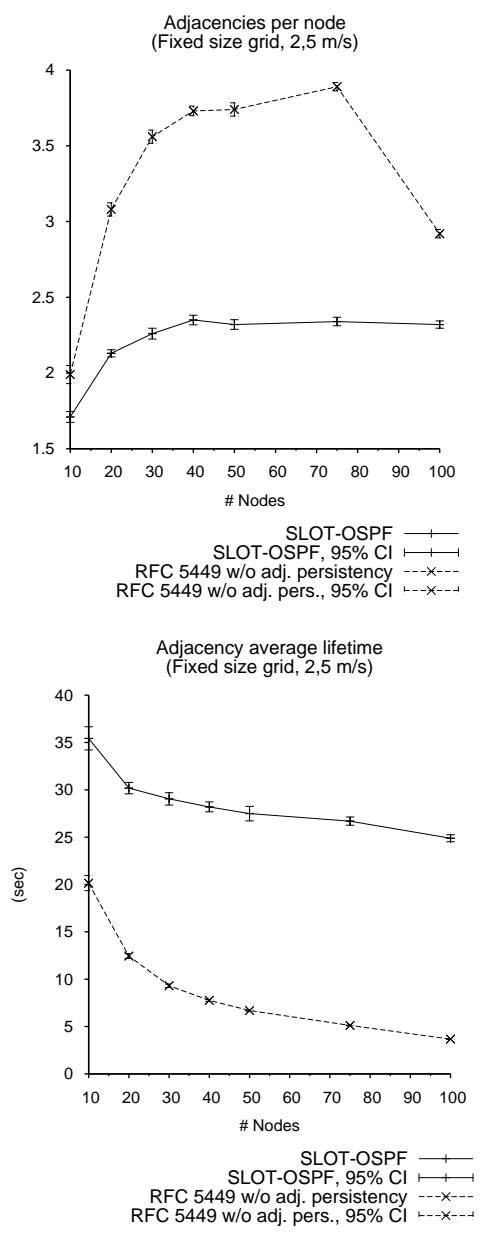

Fig. 7. Average per node number of adjacencies, and adjacency lifetime, in seconds.

In this context, in order to avoid the $\sqrt{\nu}$ term which can be heavy when the node density increases, we could use a quantized link cost. For example the cost of a link could be $m(A, B)=\lfloor\log \min \{\operatorname{Cost}(A, B), \operatorname{Cost}(B, A)\}\rfloor$, and the ties would be broken with IDs, as with uniform link cost. In this case we may expect a per node synchronization rate that is much smaller than $O(s \sqrt{\nu})$, closer to a constant synchronization rate. Our conjecture is that the per node synchronization rate with this refinement would be $O(s \log \nu)$. This opens interesting perspectives in the realm of optimized synchronized link overlay.

Future work will include proving this conjecture rigorously, and better link metric tuning in order to properly balance the synchronization rate and the per synchronization overhead. For example one can also put an upper bound such as $m(A, B)=\min \{C,\lfloor\log \min \{\operatorname{Cost}(A, B), \operatorname{Cost}(B, A)\}\rfloor\}$ for some constant $C$.

\section{REFERENCES}

[1] J. Moy: RFC 2328, OSPF Version 2. Internet Society (ISOC). April 1998.

[2] R. Coltun, D. Ferguson, J. Moy: RFC 2740, OSPF for IPv6. Internet Society (ISOC). December 1999. 
[3] P. Jacquet, P. Mulhethaler, T. H. Clausen, A. Laouiti, A. Qayyum, L. Viennot: Optimized Link State Routing for Ad Hoc Networks. HIPERCOM Project / INRIA Rocquencourt. Proceedings of the IEEE International Multitopic Conference (INMIC). 2001.

[4] A. Qayyum, L. Viennot, A. Laouiti: Multipoint Relaying for Flooding Broadcast Messages in Mobile Wireless Networks. HIPERCOM Project / INRIA Rocquencourt. Proceedings of the 35th Annual Hawaii International Conference on System Sciencias (HICSS). 2002.

[5] G. F. Riley: The Georgia Tech Network Simulator. Proceedings of the ACM SIGCOMM 2003 Workshops. August 2003.

[6] C. Adjih, E. Baccelli, T. H. Clausen, P. Jacquet, G. Rodolakis: Fish Eye OLSR Scaling Properties. Journal of Communications and Networks. 2003.

[7] T. Henderson, P. Spagnolo, G. Pei: Evaluation of OSPF MANET Extensions. Boeing Technical Report D950-10897-1. July 2005.

[8] P. Jacquet: Optimization of Point-to-point Database Synchronization via Link Overlay RNG in Mobile Ad Hoc Networks (Rapport de Recherche 6148). INRIA Rocquencourt. February 2007.

[9] E. Baccelli, P. Jacquet, D. Nguyen: Integrating VANETs in the Internet Core with OSPF: the MPR-OSPF Approach. Proceedings of the IEEE International Conference on ITS Telecommunications (ITST). June 2007.

[10] E. Baccelli, T. Clausen, P. Jacquet, D. Nguyen: OSPF Multipoint Relay (MPR) Extension for Ad Hoc Networks. IETF Request For Comments RFC 5449, February 2009.

[11] A. Roy, M. Chandra: Extensions to OSPF to Support Mobile Ad Hoc Networking. IETF Request For Comments RFC 5820, March 2010.

[12] C. Adjih, E. Baccelli, P. Jacquet: Link State Routing in Ad Hoc Wireless Networks. Proceedings of MILCOM 2003 - IEEE Military Communications Conference, vol. 22, no. 1, pp. 1274-1279, Boston, USA, Oct. 2003.

[13] E. Baccelli, F. Baker, M. Chandra, T. Henderson, J. Macker, R. White: Problem Statement for OSPF Extensions for Mobile Ad Hoc Routing. Internet Engineering Task Force (IETF). draft-baker-manet-ospf-problemstatement-00 (work in progress), 2003.

[14] GNU Zebra, www.zebra.org

[15] INRIA OSPF Extension For MANET Code www.emmanuelbaccelli.org/ospf

[16] T.M. Cover, P.E. Hart, Nearest Neighbor Pattern Classification, IEEE Transactions on Information Theory, vol. IT-13, No.1, 1967, pp.21-27.

[17] G. Toussaint, The Relative Neighborhood Graph of Finite Planar Set, Pattern Recognition 12 (4), 261268, 1980.

[18] L. Devroye, On The Expected Size of Some Graphs in Computational Geometry, Computers and Mathematics with Applications, vol. 15, pp. 53-64, 1988.

[19] P. Jacquet, Control of mobile ad hoc networks, Proceedings of ITW 2006, Uruguay, 2006.

[20] T. Henderson, P. Spagnolo, J. H. Kim, A Wireless Interface Type for $O S P F$, Proceedings of the IEEE MILCOM Conference, 2003.

[21] E. Baccelli, J.A. Cordero, P. Jacquet, Multi-Point Relaying Techniques with OSPF on Ad Hoc Networks, Proceedings of ICSNC 2009, Porto, Portugal, Sept. 2009.

[22] A. Vasan, A. Udaya Shankar, An Empirical Characterization of Instantaneous Throughput in $802.11 \mathrm{~b}$ WLANs, Department of Computer Science, University of Maryland, www.cs.umd.edu/ shankar/Papers/80211b-profile-1.pdf

[23] F. Calí, M. Conti, E. Gregori, IEEE 802.11 Wireless LAN: Capacity Analysis and Protocol Enhancement, Proceedings of INFOCOM 1998, San Francisco, Mar. 1998.

[24] J. Cartigny, F. Ingelrest and D. Simplot, RNG Relay Subset Flooding Protocols in Mobile Ad-Hoc Networks, Int. J. Found. Comput. Sci., volume 14, 2003.

[25] Boeing OSPF MANET Extension Quagga GTNets simulation code, http://hipserver.mct.phantomworks.org/ietf/ospf/, 2008.

[26] J. Wu and H.Li, On calculating connected dominating set for efficient routing in ad hoc wireless networks, Workshop on Discrete Algorithms and Methods for Mobile Computing and Communications 7-14, 1999.

[27] R. Ogier, P. Spagnolo, Mobile Ad Hoc Network (MANET) Extension of OSPF Using Connected Dominating Set (CDS) Flooding, IETF Request For Comments RFC 5614, August 2009.

[28] D. Dubhashi, A. Mei, A. Panconesi, J. Radhakrishnan, A. Srinivasan, Fast Distributed Algorithms for (Weakly) Connected Dominating Sets and Linear-Size Skeletons, Journal of Computer System Sciences, 2005.

[29] P. Spagnolo, T. Henderson, Comparison of Proposed Ospf Manet Extensions, Military Communications Conference (MILCOM), 2006.
[30] A. Qayyum, L. Viennot, A. Laouiti, Multipoint relaying for flooding broadcast messages in mobile wireless networks, Proceedings of the 35th Annual Hawaii International Conference on System Sciences (HICSS), 2002.

[31] J. Ahrenholz, P. Spagnolo, T. Henderson, E. Baccelli, P. Jacquet, T. Clausen, OSPFv2 Wireless Interface Type, Internet Engineering Task Force (IETF). draft-spagnolo-manet-ospf-wireless-interface (work in progress), 2004.

[32] J. Wu, F. Dai, Efficient Broadcasting with Guaranteed Coverage in Mobile Ad Hoc Networks, in IEEE Transactions on Mobile Computing, 2005.

\section{APPENDIX: SIMULATION ENVIRONMENT}

The following tables describe the simulation environment parameters. Table II shows the default value of the main parameters (when not explicitly mentioned in the figures). The $\alpha \in[0,1]$ parameter defines the probability of successful reception function w.r.t. the distance, $\alpha=0.5$ standing for moderately lossy channel. Further characterization of $\alpha$ is available in [7]. Tables III] and III] show the parameters specific to the configurations considered in this paper. Note that, for fairness in the comparison with SLOT-OSPF, the adjacency persistency was disabled in RFC 5449. The code used for RFC 5614 simulations is the code developed by Boeing, available in [25], set with LSAFullness=1.

TABLE II

General Simulation Parameters.

\begin{tabular}{|c|c|}
\hline Name & Value \\
\hline \multicolumn{2}{|c|}{ Experiment Statistic Parameters } \\
\hline Seed & 0 \\
\hline Samples/experiment & 20 \\
\hline \multicolumn{2}{|c|}{ Traffic Pattern } \\
\hline Type of traffic & CBR UDP \\
\hline Packet size & 1472 bytes \\
\hline Packet rate & 85 pkts/sec \\
\hline Traffic rate & $1 \mathrm{Mbps}$ \\
\hline \multicolumn{2}{|r|}{ Scenario } \\
\hline Mobility & Random waypoint model \\
\hline Speed & $v=0,5,10,15 \frac{\mathrm{m}}{\mathrm{s}}$ (constant) \\
\hline Grid shape and size & Square, $400 \mathrm{~m} \times 400 \mathrm{~m}$ \\
\hline Radio range & $150 \mathrm{~m}$ \\
\hline Wireless $\alpha$ & 0.5 \\
\hline Pause time & $40 \mathrm{sec}$ \\
\hline MAC protocol & IEEE $802.11 \mathrm{~b}$ \\
\hline \multicolumn{2}{|c|}{ OSPF General Configuration } \\
\hline HelloInterval & $2 \mathrm{sec}$ \\
\hline DeadInterval & $6 \mathrm{sec}$ \\
\hline RxmtInterval & $5 \mathrm{sec}$ \\
\hline MinLSInterval & $5 \mathrm{sec}$ \\
\hline MinLSArrival & $1 \mathrm{sec}$ \\
\hline
\end{tabular}

TABLE III

RFC 5449 AND SLOT-OSPF SPECIFIC PARAMETERS.

\begin{tabular}{|c|c|}
\hline Name & Value \\
\hline \hline AckInterval & $1800 \mathrm{msec}$ \\
\hline Flooding MPR? & Yes \\
\hline Topology Reduction & MPR Topology Reduction \\
\hline Adjacency Selection & MPR or SLOT Adjacency Reduction \\
\hline Adjacency Persistency & Disabled \\
\hline
\end{tabular}

